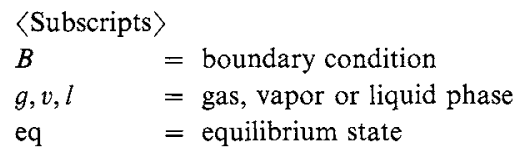

\section{Literature Cited}

1) Benedict, M., T. Pigford and H. Levi: "Nuclear Chemical Engineering," 2nd ed., Chapter 13, McGraw-Hill (1981).

2) Butler, J. P.: "Separation of Hydrogen Isotopes," p. 93, ACS Symp. Ser. 68, ACS (1978).

3) Butler, J. P., et al.: Proc. Sixth Int. Congr. Catalysis, London 1976, B14.

4) Cohen, K.: "Theory of Isotope Separation," p. 126, McGrawHill (1957).
5) Enright, J. T. and K. T. Chuang: Can. J. Chem. Eng., 56, 246 (1978).

6) Hodor, I., M. Peculea, R. Strāulea: Isotopenpraxis, 9, 6 (1973).

7) Kitamoto, A., Y. Takashima and M. Shimizu: 2nd World Congr. Chem. Engng, Montreal 1981, 7-3-5.

8) London, H.: "Separation of Isotopes," p. 95, George Newnes (1961).

9) Mercea, V.: Énergie Nucléaire, 10, 367 (1968).

10) Rae, H. K.: "Separation of Hydrogen Isotopes," p. 1, ACS Symp. Ser. 68, ACS (1978).

11) Rolston, J. H. et al.: J. Phys. Chem., 80, 1064 (1976).

12) Shimizu, M., A. Kitamoto and Y. Takashima: J. Nucl. Sci. Technol., 20, 36 (1983).

13) Stevens, W. H.: Can. Pat. Appl., No. 119,402 (1971).

\title{
A STUDY OF THE INITIAL STAGE OF OIL SPREADING ON CALM WATER THROUGH METHODS OF BOTH INSTANTANEOUS AND CONSTANT RATE OF OIL RELEASE
}

\author{
TETSUO AKIYAMA, SATORU MITSUMORI, TORU TERADA \\ AND KOZO KOIDE \\ Department of Chemical Engineering, Shizuoka University, Hamamatsu 432
}

\begin{abstract}
The initial stage of oil spreading on calm water was investigated for one-dimensional flow. Two kinds of experimental methods, instantaneous and constant rate of oil release, were used to investigate the rate of spreading and its dependence on surface contamination. Experimental and theoretical results were compared and some apparent discrepancies, hitherto unreported, are reported here. Also, a modified empirical correlation was obtained to express the spreading rate of oil slicks in the case of instantaneous oil release.
\end{abstract}

\section{Introduction}

The task of predicting how oil spreads in calm water was first dealt with in a systematic way by Fay, ${ }^{2)}$ who has shown by essentially dimensional analysis that there are three stages in the spreading of an initially concentrated volume of oil: gravityinertial, gravity-viscous and surface tension-viscous spreading regimes. Shudo ${ }^{6}$ extended the analysis to the case when oil is released at a constant rate. The analysis of oil spreading has been advanced considerably by Hoult ${ }^{4)}$ and Fannelop and Waldman ${ }^{3)}$ among others.

Surface contaminants are also known to affect the spreading behavior of oil spills. Unno and Inoue ${ }^{8)}$ carried out experiments to investigate the effect of surface contaminants on the spreading rate of soybean oil. Recent developments regarding the gravity current are reviewed by Simpson. ${ }^{5}$

The objectives of the present study were first, to

Received November 12, 1982. Correspondence concerning this articie should be addressed to T. Akiyama. extend the study of the previous work ${ }^{11}$ for oils of a broader range of physical properties and to obtain a modified correlation to express the spreading rate of oil on calm water; and second, to study the constant rate of oil release both theoretically and experimentally. It was reported in the previous work that the length of the water channel had a significant influence on the spreading rate of oil, which seemed to have been caused by the disturbance that occurred when the shutter was lifted. To avoid the effect of this disturbance, the method of constant rate of oil release was tried in this study. Some disagreements were found between the theoretical and experimental results in the case of constant rate of oil release. In an attempt to find the cause of the disagreements some experiments were carried out to find the effect of surface contamination, and some studies were made to determine the dependence of the rate of spreading on thickness of the oil film.

\section{Theoretical Analysis}

Theoretical analysis for the case of instantaneous 
release has been advanced by several authors. ${ }^{2-4)}$ Shudo's work ${ }^{6)}$ for the constant rate of oil release depends only on the dimensional analysis by Fay. ${ }^{2)}$ In this report we followed closely the analysis by Fannelop and Waldman, ${ }^{3)}$ and solved equations of motion and continuity. Details are shown in Appendix.

\section{Experiments}

Experiments were conducted at $293.2 \mathrm{~K}$ using tap water and the following oils-kerosene, light oil, liquid paraffin, motor oil MS-30, salad oil and turpentine oil. Physical properties of these oils, together with corrected values of the interfacial tension for motor oil MS-30 and super oils A, B, C, D and E (which were used in the previous work) ${ }^{1}$ are listed in Table 1. We found that the Du Noüy tensiometer was not quite dependable, and the Traubes drop measuring method was used to correct values of interfacial tension in the previous work. ${ }^{1)}$ The Du Noüy tensiometer measures interfacial tension by expanding the liquid film until it breaks down, so that this tensiometer does not seem suitable to deal with fluids that contain volatile matter, whereas the Traubes drop method is not so sensitive to volatile matter and seems suited to deal with it. As an additional property the spreading coefficient is included in the table. Experimental apparatus was essentially the same as that reported in the previous work. ${ }^{1)}$ Two vessels made of acrylic resin (length, width and depth are $1.00 \times 0.15 \times 0.09 \mathrm{~m}$ and $1.80 \times 0.15 \times 0.19 \mathrm{~m}$ ) were used as water channels. A feed system was added in experiments for the constant rate of oil release. After every run vessels were washed three times with powder soap, then washed thoroughly with running water. This procedure enabled us to obtain reproducible results.

\subsection{Constant rate of oil release}

Experimental apparatus was composed of water channels and an oil feeder. The arrangement is shown in Fig. 1. Oil was allowed to flow gently down from the head tank into the region between the wall and the dam, and then oil started to overflow over the dam and spread on calm water. To see local oil slick velocities, tiny pieces of aluminum foil and styrofoam were used as tracers on the oil surface. Three kinds of oils, motor oil MS-30, light oil, and salad oil, were used for these experiments. Photographs were taken from above.

\subsection{Instantaneous oil release}

Basically the same experimental procedures as reported in the previous work were followed. The larger water channel was filled with tap water at $293.2 \mathrm{~K}$. Oil was gently poured in the region between the wall and the shutter. After the oil settled, the shutter was removed instantaneously. To determine the effect of surface contamination with motor oil MS-30, an
Table 1. Physical properties of oils (at $293.2 \mathrm{~K}$ )

\begin{tabular}{lcccr}
\hline \multicolumn{1}{c}{ Materials } & Density & Viscosity & $\begin{array}{c}\text { Interfacial } \\
\text { tension }\end{array}$ & $\begin{array}{r}\text { Spreading } \\
\text { coefficient }\end{array}$ \\
& $\sigma\left[\mathrm{kg} \cdot \mathrm{m}^{-3}\right]$ & $\mu[\mathrm{Pa} \cdot \mathrm{s}]$ & $\sigma\left[\mathrm{N} \cdot \mathrm{m}^{-1}\right]$ & $\bar{\sigma}\left[\mathrm{N} \cdot \mathrm{m}^{-1}\right]$ \\
\hline MS-30 & 889.3 & 0.2756 & 0.0200 & 0.0186 \\
Super oil A & 871.8 & 0.0689 & 0.0486 & -0.0083 \\
Super oil B & 883.7 & 0.1458 & 0.0498 & -0.0098 \\
Super oil C & 887.8 & 0.2175 & 0.0578 & -0.0187 \\
Super oil D & 890.3 & 0.4986 & 0.0621 & -0.0235 \\
Super oil E & 894.3 & 0.9837 & 0.0630 & -0.0250 \\
Kerosene & 787.6 & 0.0017 & 0.0461 & 0.0007 \\
Light oil & 826.1 & 0.0045 & 0.0308 & 0.0146 \\
Liquid paraffin & 874.6 & 0.2284 & 0.0632 & -0.0240 \\
Salad oil & 915.4 & 0.0668 & 0.0244 & 0.0126 \\
Turpentine oil & 857.5 & 0.0015 & 0.0207 & 0.0252 \\
\hline
\end{tabular}

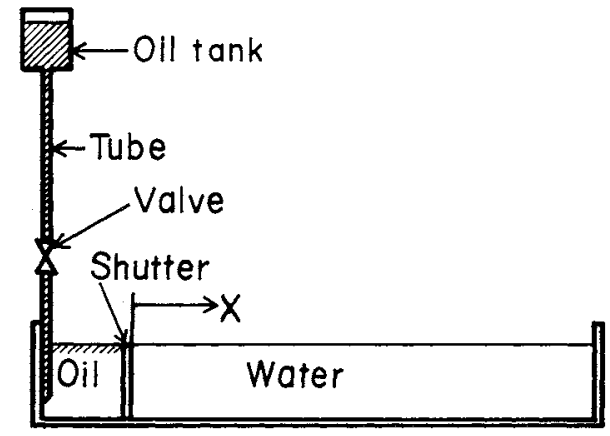

Fig. 1. Experimental apparatus for constant rate of oil release.

additional step was introduced. That is, a small quantity of MS-30 was spread uniformly over the water to form a very thin film of oil before it was released instantaneously by lifting the shutter.

\section{Results and Discussion}

\subsection{Constant rate of oil release}

Two stages of oil spreading of motor oil MS-30 are shown in Fig. 2. A thin oil film (first stage) was observed to start spreading over the dam before another oil film of finite thickness (second stage) started to spread. Then both of these oil slicks spread together at their own speeds. The thin oil film spread until it reached the opposite end of the vessel. Vessel length did not affect the spreading velocity. This is quite a contrast to the experimental finding in the case of instantaneous oil release. It was reported in the earlier work ${ }^{1)}$ that the disturbance caused by lifting the shutter seemed to have caused a deviation of the smaller vessel's data from those of the larger one at an early time. In the first stage the spreading rate was not dependent on the flow rate, as is illustrated in Fig. 3; therefore, it can be considered to be in the surface tension-viscous regime. In experiments with light oil and salad oil, similar results were obtained except that the spreading in the second stage was not so clearly discernible. Using the dimensionless coordinates by 


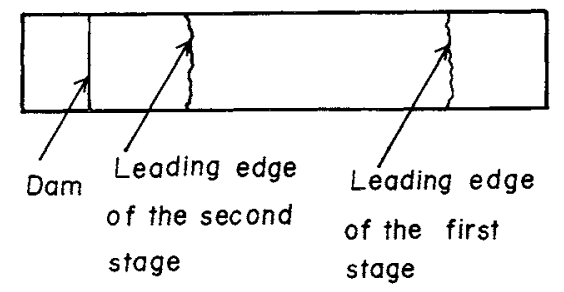

Fig. 2. Two stages of oil spreading.

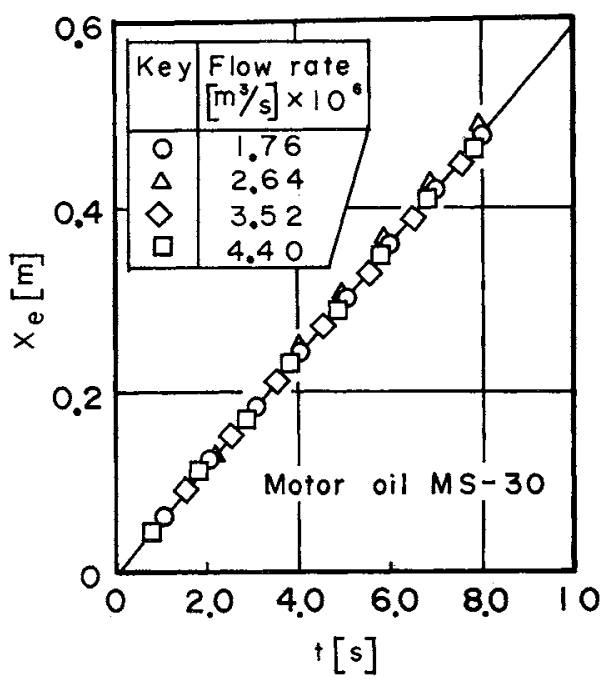

Fig. 3. Leading edge of oil slicks in first stage vs. time.

Takahashi et al., ${ }^{6)}$ the rate of the spreading in the first stage is expressed in Fig. 4. The straight line represents the following relationship:

$$
x_{\mathrm{e}}=2.5 \times 10^{-3}\left(\bar{\sigma} / \mu_{\mathrm{w}}\right) t
$$

The range of applicability of the above equation is for

$$
3 \times 10^{6}<t \rho_{\mathrm{w}} \bar{\sigma} / \mu^{3}<3 \times 10^{9}
$$

The exponent of $t$ in Eq. (1) coincides with the theoretical result for the gravity-inertial regime, Eq. (A-11), rather than Eq. (A-19) which represents the surface tension-viscous regime. No clear-cut explanation has been found for this apparent discrepancy yet.

In the second stage the rate of spreading is as shown in Fig. 5. It shows that $x_{\mathrm{e}}$ is proportional to $q^{1 / 3}$ and $t$, which coincides with the theoretical results for the gravity-inertial regime, Eqs. (A-11) and (A16). The straight line in the figure can be expressed by the formula

$$
x_{\mathbf{e}}=0.350(g \cdot q / w)^{1 / 3} t
$$

where the range of $q$ was from $1.76 \times 10^{-6} \mathrm{~m}^{3} / \mathrm{s}$ to $4.40 \times 10^{-6} \mathrm{~m}^{3} / \mathrm{s}$. Shown in Fig. 6 are the loci of tracers on the spreading oil slick. This figure displays the relative movement of the first- and second-stage oil slicks, and also indicates that the rate of spreading is approximately constant regardless of starting po-

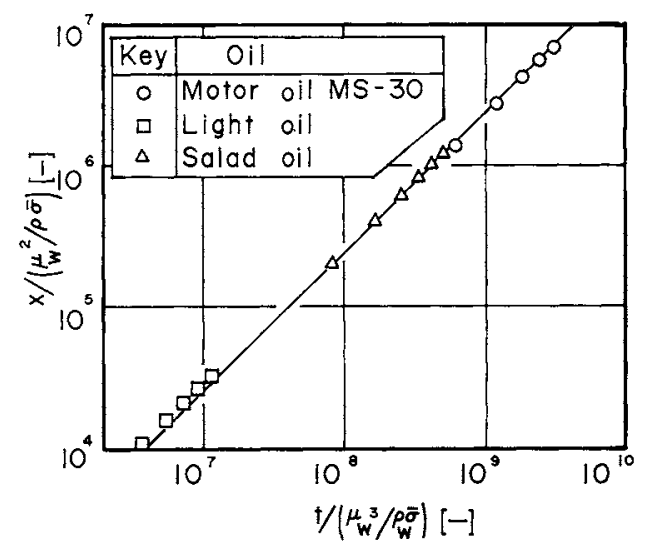

Fig. 4. Leading edge of oil slicks in first stage vs. time expressed in dimensionless form.

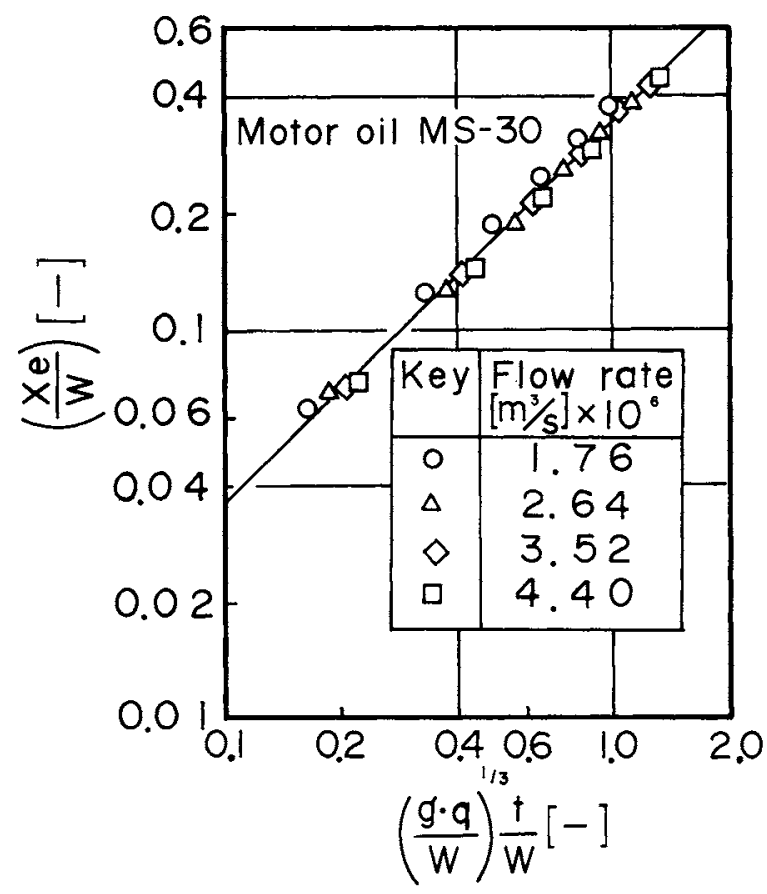

Fig. 5. Leading edge of oil slicks in second stage vs. time.

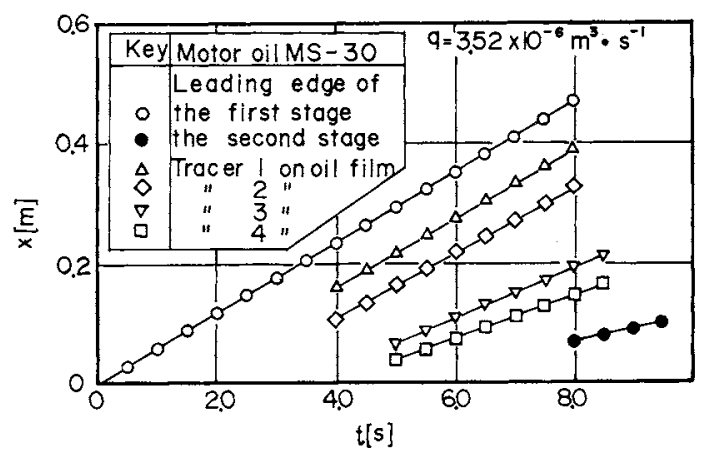

Fig. 6. Locus of tracers on oil slick.

sition, but that the rate is increasingly large the nearer the position is to the leading edge. Applying the theoretical results of the gravity-inertial regime for the second stage of spreading, we obtained $A=2.167 \times$ 
$10^{-2} \mathrm{~m} / \mathrm{s}, \quad \delta=1.08 \times 10^{-3} \mathrm{~m}$ when $q=3.52 \times 10^{-6}$ $\mathrm{m}^{3} / \mathrm{s}$. Now, Fannelop and Waldman ${ }^{3)}$ assumed, in the course of deriving the formula for the spread of an oil slick, that the velocity of the leading edge is proportional to the characteristic wave speed for a small disturbance, i.e.,

$$
u_{\mathrm{e}}=\frac{\mathrm{d} x_{\mathrm{e}}}{\mathrm{d} t}=k\left(\alpha \delta_{\mathrm{e}}\right)^{1 / 2}
$$

where $k$ is the proportional constant. Fannelop and Waldman set $k=1$, which compared favorably with the experimental work by Hoult et al. ${ }^{4)}$ To see if this applies in our second stage of spreading the above equation was used to calculate the spreading velocity for given $\delta=1.08 \times 10^{-3} \mathrm{~m}$. The velocity calculated was $3.46 \times 10^{-2} \mathrm{~m} / \mathrm{s}$, which is much larger $(54.8 \%)$ than the experimental value of $2.167 \times 10^{-2} \mathrm{~m} / \mathrm{s}$. Surface contamination due to the oil spreading in the first stage might have affected the spreading velocity of the second stage. Thus, to determine the effect of contamination due to the prespreading, MS-30 was spread on the water before constant flow began. Shown in Fig. 7 is the effect of the prespreading. The spreading velocity was slowed down by about $30 \%$ compared to that on clean water due to the prespreading. The prespread oil amounted to an oil film of $3 \mu \mathrm{m}$ thickness. This oil-film thickness was calculated by dividing the quantity of prespread oil by the surface area of the water channel. Further application of the prespreading oil did not alter the spreading rate. Furthermore, no film of spreading oil in the first stage was observed in this case. An oil slick of a finite thickness was observed from the very beginning. Therefore, surface contamination might be a factor in the decrease of the spreading velocity for a given thickness. Other conceivable causes for the decrease of the spreading velocity may be that the thickness of the oil slick was too thin. It is also clear from Eqs. (A17) and (A-18) that the theoretical results of the gravity-viscous regime fail to explain the phenomena. In any case, the theoretical and experimental results of neither the first nor the second stage of the oil spreadings quite agreed, and further work seems needed to study more fully the physical structures of the spreading of multicomponent oil.

\subsection{Instantaneous oil release}

Experimental results, including those of the previous work, ${ }^{1)}$ are shown in Fig. 8 along with a modified empirical correlation

$$
x_{\mathrm{e}} / L=0.896\left[\left(g \cdot \delta_{\mathrm{i}}\right)^{1 / 2}\left(\mu_{0} / \sigma\right)\right]^{-0.073} \cdot\left[(\alpha / L)^{1 / 2} t\right]^{0.87}
$$

Note that the definition of the coordinate $x$ is slightly different from the previous one (see Fig. 1). The fact that $x_{\mathrm{e}}$ is proportional to $t^{0.87}$ is the same as before, but other coefficients have been changed somewhat due to the fact that the values of surface tension in the

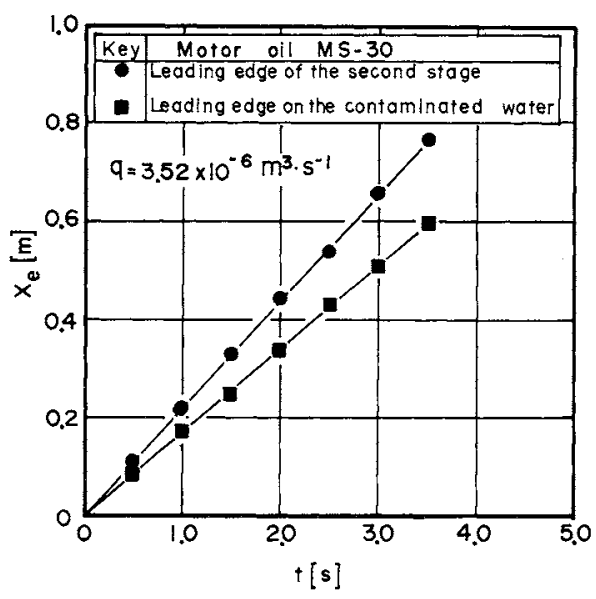

Fig. 7. Leading edge of oil slicks on contaminated water surface vs. time for constant rate of oil release.

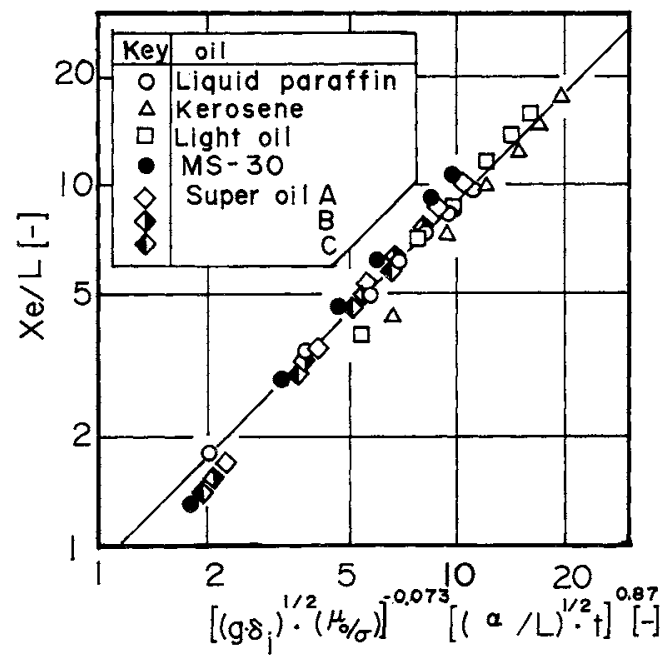

Fig. 8. Leading edge of oil slicks vs. dimensionless time for instantaneous oil release.

previous work have been corrected, and also a broader range of new data was added to formualte the correlation. The data were also compared to the correlation by Takahashi et al $^{7)}$ Most of our data lay in the range of dimensionless time between zero and one, while most of their data lay in the range of dimensionless time greater than one, and meaningful comparisons could not be made. More fundamentally, the super oils A-E as well as liquid paraffin have a negative spreading coefficient (see Table 1), which makes it impossible to use the dimensionless time by Takahashi et al. ${ }^{77}$ In fact, the super oils as well as liquid paraffin spread uniformly at an earlier time but then scattered into irregular forms as time elapsed. The reason why our data lay in a small range of time has been mentioned in the previous work, i.e., the reflection of the water wave seemed to have delayed the rate of oil spreading by a few seconds.

Effects of surface contamination The motor oil MS30 was used to determine the effect of the prespread- 
ing. The prespread oil covered the water surface uniformly. Experimental results showing the effect of the prespreading are shown in Fig. 9, wherein the prespread thickness of the oil film, $\delta$, was used as a parameter. Another illustration is Fig. 10, which shows the dependence of the spreading rate on the degree of contamination. These figures clearly demonstrate that the spreading rate is dependent on time and that the rate is progressively reduced as the thickness of the film of prespread oil increases until it reaches approximately $3 \mu \mathrm{m}$. Thereafter, the rate of spreading ceases to depend on film thickness.

It is interesting to note that the oil lies on the free water surface as a monolayer of an approximate thickness of $2.5 \times 10^{-3} \mu \mathrm{m}$, which is the length of the hydrocarbon chains. Unno and Inoue ${ }^{7)}$ measured the spreading pressure for olive oil. They observed a remarkable effect of the oil film's thickness on the spreading pressure. It decreased sharply as the film's thickness increased until the thickness reached $1 \times 10^{-2} \mu \mathrm{m}$. Thereafter, the rate of decrease slacked and leveled off at about $5 \times 10^{-2} \mu \mathrm{m}$. This thickness is about twenty times greater than that of the monolayer hydrocarbon chains, but sixty times smaller than $3 \mu \mathrm{m}$ (beyond which the rate of spreading ceased to depend on film thickness in this study). The difference in these values may be caused by the wall effects or the different oils used. Furthermore, it appears that the effect of each constituent in an oil must be taken into account when the oil consists of more than one component.

\section{Conclusions}

Experiments in one-dimensional oil spreading on calm water were carried out through methods of both instantaneous and constant rate of oil release. For constant rate of oil release, the similarity solution was considered, and it was found that the theoretical and experimental rates of the oil spreading did not quite agree in the surface tension-viscous regime. The law of the characteristic wave speed for small distrubance did not seem to apply for the gravity-inertial regime over the experimental range investigated. The cause for this disagreement has yet to be found.

In the case of instantaneous release of oil, a modified empirical correlation covering 11 kinds of oils was obtained to express the spreading rate of oil slicks. The effect of surface contamination, due to the prespreading of the same oil, was investigated for motor oil MS-30. It was found that the rate of spreading is dependent on the degree of contamination on the water surface.

\section{Appendix}

The following similarity variable is used:

$$
X=x / x_{\mathbf{e}}
$$

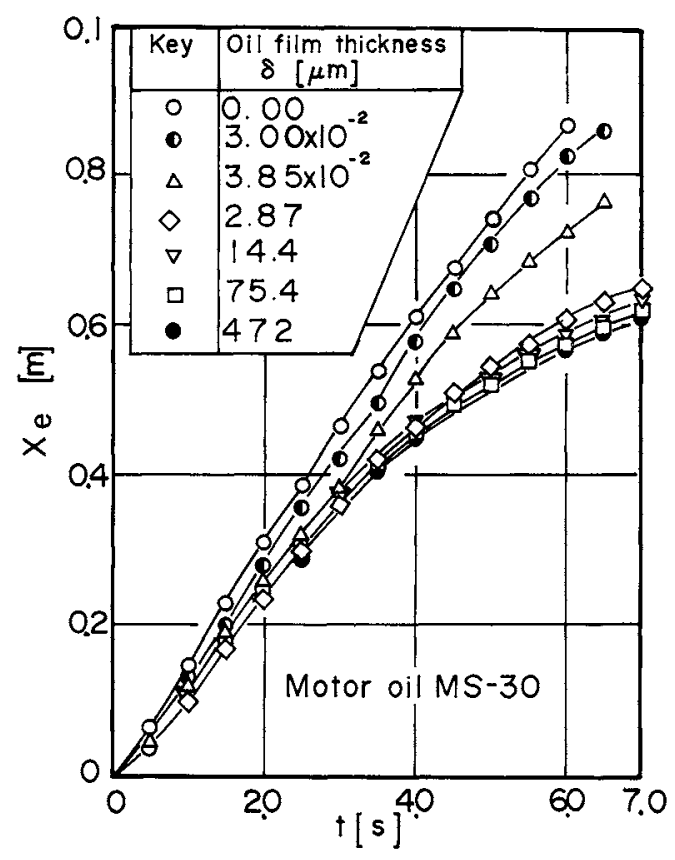

Fig. 9. Leading edge of oil slicks on contaminated water surface vs. time.

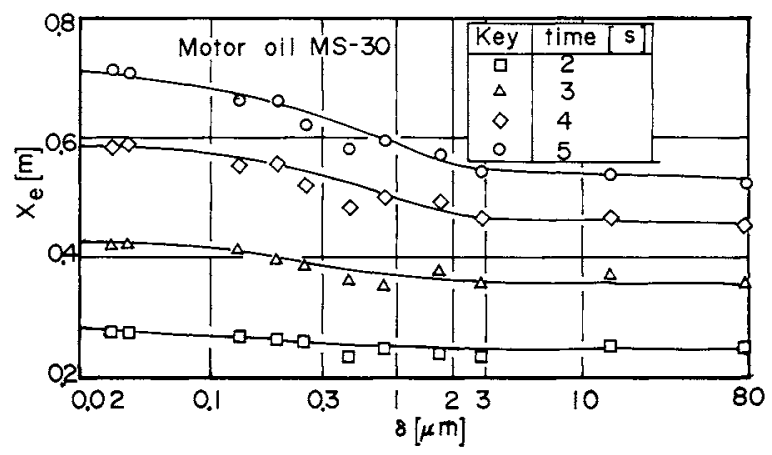

Fig. 10. Leading edge of oil slicks on contaminated water surface vs. thickness of oil slick.

where

$$
x_{\mathrm{e}}=A t^{n}
$$

which represents the position of the leading edge of oil. The $A$ and $n$ are constants which vary depending on the regime.

The equation of motion for unidirectional oil spreading is ${ }^{3)}$

$$
\frac{\partial u}{\partial t}+u \frac{\partial u}{\partial x}=-\alpha \frac{\partial \delta}{\partial x}-\tau_{\mathrm{w}} / \rho_{\mathrm{o}} \delta
$$

The equation of continuity is

$$
\frac{\partial \delta}{\partial t}+u \frac{\partial \delta}{\partial x}+\delta \frac{\partial u}{\partial x}=0
$$

where

$$
\alpha=g \cdot\left(1-\rho_{\mathrm{o}} / \rho_{\mathrm{w}}\right)
$$

All the symbols are summarized in the nomenclature. The constant flow rate implies that

$$
w \int_{0}^{x_{\mathrm{e}}} \delta \mathrm{d} x=q t
$$

To obtain a similarity solution $\delta$ must satisfy the following formula: 


$$
\delta=D(X) t / x_{\mathrm{e}}
$$

where $D(X)$ is subject to the condition

$$
\int_{0}^{1} D(X) \mathrm{d} X=q / w
$$

Since Eq. (2) gives $u\left(x_{\mathrm{e}}\right)=A n t^{n-1}, u(x)$ becomes, to satisfy the condition,

$$
u=U_{\mathrm{o}}(X) t^{n-1}
$$

where $U_{\mathrm{o}}(X)$ is a function only of $X$. To obtain a similarity solution it becomes necessary to divide the region into subregions.

Gravity-inertial regime Neglecting the term $\tau_{\mathrm{w}}$, and substituting Eqs. (A-6) and (A-8) into Eqs. (A-3) and (A-4), we have

$$
\begin{aligned}
& \left(\frac{U_{0}}{A}-2 X\right) \frac{\mathrm{d} U_{0}}{\mathrm{~d} X}+\frac{\alpha}{A^{2}} \frac{\mathrm{d} D}{\mathrm{~d} X}=0 \\
& \left(\frac{U_{0}}{A}-2 X\right) \frac{\mathrm{d} D}{\mathrm{~d} X}+\frac{D}{A} \frac{\mathrm{d} U_{0}}{\mathrm{~d} X}=0
\end{aligned}
$$

and

$$
x_{\mathrm{e}}=A t
$$

The above equations reveal a significant difference between constant-rate oil release and instantaneous oil release. In the latter, a unique solution $U_{\mathrm{o}}(X)$ can be obtained solely from the equation of continuity, and $D(X)$ can be derived from the equation of motion, whereas Eqs. (A-9) and (A-10) must be solved simultaneously for constant-rate oil release.

Multiplying Eq. (A-9) by $\mathrm{d} D / \mathrm{d} X$ and Eq. (A-10) by $\mathrm{d} U_{0} / \mathrm{d} X$ and subtracting we have

$$
\left(\frac{\alpha}{A}\right)\left(\frac{\mathrm{d} D}{\mathrm{~d} X}\right)^{2}=D\left(\frac{\mathrm{d} U_{0}}{\mathrm{~d} X}\right)^{2}
$$

From which we obtain

$$
U_{\mathrm{o}}=2 \sqrt{\alpha D / A}+\beta
$$

where $\beta$ is an integration constant. Substituting the above relations into Eq. (A-10) we get either

$$
D=\text { constant }
$$

or

$$
D=A(2 A X-\beta)^{2} / 9 \alpha
$$

If the latter relationship holds we get, from Eq. (A-13),

$$
U_{\mathrm{o}}=(4 A X+\beta) / 3
$$

From Eq. (A-2), $\beta=-A$. Then it follows from the above equation that $U_{0}(0)=-A / 3$, which contradicts the physical reality. When $D$ is constant $U_{\mathrm{o}}$ becomes constant also, and the similarity solutions for $U$ and $\delta$ become

$$
\begin{gathered}
U=A \\
\delta=D / A
\end{gathered}
$$

where $D$ is constant here. This leads to

$$
A \propto q^{1 / 3}
$$

The above results coincide with those of Shudo's dimensional analysis.
Gravity-viscous regime No analytical solution seems obtainable for the velocity profile in this regime, and we only present the expression for the leading edge.

$$
\begin{gathered}
x_{\mathrm{e}}=A t^{7 / 8} \\
A \propto q^{1 / 2}
\end{gathered}
$$

Surface tension-viscous regime Since the rate of spread in this regime is independent of the volume of the spill, the identical result with that of the instantaneous release will apply. Thus we have ${ }^{3)}$

$$
x_{\mathrm{e}}=(4 \pi / 3)^{1 / 2}\left[\bar{\sigma}^{1 / 4} /\left(\rho_{\mathrm{w}} \mu_{\mathrm{w}}\right)^{1 / 4}\right] t^{3 / 4}
$$

where $\bar{\sigma}$ refers to the spreading coefficient.

\section{Nomenclature}

A $\quad=$ coefficient in Eq. (A-2) [m. $\left.\mathrm{s}^{-n}\right]$

$D \quad=$ defined by Eq. (A-6) $\left[\mathrm{m}^{2} \cdot \mathrm{s}^{-1}\right]$

$g \quad=$ gravity $\left[\mathrm{m} \cdot \mathrm{s}^{-2}\right]$

$k \quad=$ constant in Eq. (3) $\quad[-]$

$L \quad=$ characteristic length; (initial oil volume $/ w)^{1 / 2}$

[m]

$\begin{array}{llr}n & =\text { constant in Eq. (A-2) } & {[-]} \\ q & =\text { flow rate } & {\left[\mathrm{m}^{3} \cdot \mathrm{s}^{-1}\right]}\end{array}$

$\begin{array}{llr}n & =\text { constant in Eq. (A-2) } & {[-]} \\ q & =\text { flow rate } & {\left[\mathrm{m}^{3} \cdot \mathrm{s}^{-1}\right]}\end{array}$

$q \quad=$ time $t \quad[\mathrm{~s}]$

$u \quad=$ velocity $\left[\mathrm{m} \cdot \mathrm{s}^{-1}\right]$

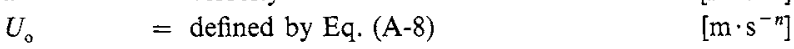

$w \quad=$ channel width $\quad[\mathrm{m}]$

$x \quad=$ coordinate as shown in Fig. $1 \quad$ [m]

$X \quad=x / x_{\mathrm{e}}$

$\alpha \quad=g\left(1-\rho_{0} / \rho_{\mathrm{w}}\right) \quad\left[\mathrm{m} \cdot \mathrm{s}^{-2}\right]$

$\beta \quad=$ constant in Eq. (13) $\quad\left[\mathrm{m} \cdot \mathrm{s}^{-1}\right]$

$\delta \quad=$ oil-film thickness $\quad[\mathrm{m}]$

$\mu \quad=$ viscosity $\quad[\mathrm{Pa} \cdot \mathrm{s}]$

$\rho \quad=$ density $\quad\left[\mathrm{kg} \cdot \mathrm{m}^{-3}\right]$

$\sigma \quad=$ interfacial tension between water and $\quad\left[\mathrm{N} \cdot \mathrm{m}^{-1}\right]$

$\bar{\sigma} \quad=$ spreading coefficient $\quad\left[\mathrm{N} \cdot \mathrm{m}^{-1}\right]$

$\tau \quad=$ shear stress $\quad[\mathrm{Pa}]$

〈Subscripts〉

$\begin{array}{ll}\mathrm{e} & =\text { leading edge } \\ \mathrm{i} & =\text { initial } \\ \mathrm{o} & =\text { oil } \\ \mathrm{W} & =\text { water }\end{array}$

\section{Literature Cited}

1) Akiyama, T., T. Nishioka, H. Baba, S. Mitsumori and K. Koide: J. Chem. Eng. Japan, 13, 417 (1980).

2) Fay, J. A.: "Oil on the Sea" (ed. by Hoult, D. P.), Plenum, New York-London (1969).

3) Fannelop, T. K. and G. D. Waldman: AIAA J., 10, 506 (1972).

4) Hoult, D. P.: Ann. Rev. Fluid Mech., 4, 341 (1972).

5) Simpson, J. E.: Ann. Rev. Mech., 14, 213 (1982).

6) Shudo, N.: Third Kankyomondai Symposium Lecture Note, Doboku Gakkai, 82 (1975).

7) Takahashi, T., Y. Kitamura and K. Nakada: Kagaku Kogaku Ronbunshu, 5, 526 (1979).

8) Unno, H. and I. Inoue: Chem. Eng. Sci., 33, 1425 (1978). 